www.jmscr.igmpublication.org

Impact Factor (SJIF): 6.379

Index Copernicus Value: 71.58

ISSN (e)-2347-176x ISSN (p) 2455-0450

crossrefDOI: https://dx.doi.org/10.18535/jmscr/v6i7.125

Journal Of Medical Science And Clinical Research

\title{
Radiotherapy in Post Mastectomy High Risk Breast Cancer: Early results of a Prospective Study comparing Conventional versus Hypofractionated Radiotherapy
}

\author{
Authors \\ Apurba Kumar Kalita ${ }^{1}$, Mouchumee Bhattacharyya ${ }^{1}$, Vikas Kantilal Jagtap ${ }^{2}$ \\ Rubu Sunku ${ }^{1}$, Partha Pratim Medhi ${ }^{1}$, Fahim Arman Hasan ${ }^{3}$, Shubhalakshmi Saikia ${ }^{1}$, \\ Amal Chandra Kataki ${ }^{4}$ \\ ${ }^{1}$ Department of Radiation Oncology, Dr. B. Borooah Cancer Institute, Guwahati, Assam, India \\ ${ }^{2}$ Department of Radiation Oncology, NEIGRIHMS, Shillong, Meghalaya, India \\ ${ }^{3}$ Department of Radiation Oncology, State Cancer Institute-GMCH, Guwahati, Assam, India \\ ${ }^{4}$ Director, Dr. B. Borooah Cancer Institute, Guwahati, Assam, India \\ Corresponding Author
}

Partha Pratim Medhi, MD (Radiotherapy)

Registrar, Department of Radiation Oncology, Dr. Bhubaneswar Borooah Cancer institute, Gopinath Nagar,

Guwahati-781016, Dist.- Kamrup (M), Assam, India

Phone no.: +918399815602, Email: parthapmedhi88@gmail.com

\begin{abstract}
Purpose: Role of adjuvant radiotherapy postmastectomy in improving local-regional control and overall survival is well established. Conventional radiotherapy delivers 50-60 Gray (Gy) in 5-6 weeks time and often women find it difficult to comply with this treatment. A shorter duration hypofractionated treatment, if found to be safe and effective, will be more convenient for patients and treatment providers alike and hence the need for this prospective study

Materials \& Methods: Fifty breast cancer patients who underwent mastectomy were enrolled and randomized into the study arms- Conventional Arm (CF) [50Gy in 25 Fractions] and Hypofractionation arm $(H F)$ [40Gy in 15 Fractions]. Treatment related acute toxicities and compliance to treatment were recorded and compared between the arms using appropriate statistical tools.

Results: Both arm enrolled 25 patients each and their baseline characters were found well matched. Incidence of Grade2 and 3 acute skin toxicities was higher in the CF arm (28\% and 8\%, respectively) as compared to $\mathrm{HF}$ arm (16\% and $0 \%$, respectively). Grade 1 Odynophagia was also higher in CF arm (68\% vs $52 \%$ ). The difference in toxicities was not statistically significant ( $p$-value >0.05). With regards to treatment duration, a mean delay of 1.8 days in HF arm versus 4.76 days in CF arm was observed ( $p$ value 0.0009), implying that the shorter duration HF arm had significantly less interruptions in treatment completion.

Conclusion: In postmastectomy setting, hypofractionated radiotherapy showed lesser acute toxicities, was better in terms of treatment compliance and hence can be used routinely.

Keywords: Hypofractionation, Postmastectomy, Breast Cancer, Radiotherapy.
\end{abstract}




\section{Introduction}

Breast cancer is the most frequently diagnosed cancer in females, representing one in four of all cancers in women and is also the leading cause of cancer death among females worldwide ${ }^{1}$. Multimodality approach is required to cure this dreaded disease with radiotherapy playing an important role. An updated result ${ }^{2}$ from the Danish $82 b^{3}$ and $82 \mathrm{c}^{4}$ trials have shown that addition of locoregional RT in breast cancer patients significantly decreased the probability of local recurrence $(14 \%$ vs $49 \%$ ), distant metastasis (53\% vs 64\%) and any breast cancer related event (59\% vs $73 \%$ ) at 18 years follow up. Early Breast Cancer Trialists' Collaborative Group (EBCTCG) $)^{5}$ meta-analysis of 78 prospective randomized clinical trials demonstrated the relationship between the absolute reduction in 5-year local recurrence risk and breast cancer mortality. They reported that, if Post Mastectomy Radiotherapy (PMRT) reduced the 5-year local recurrence risk by $20 \%$, the 15 year breast cancer mortality rate would be reduced by $5.2 \%$, suggesting that the addition of PMRT would eliminate one breast cancer death at 15 years for every four local recurrences prevented. Conventional fractionation radiotherapy (CFRT) delivers 50-60 Gy at 2.0 Gy per fraction over 5-6 weeks time. However, women find it difficult to comply with such a lengthy duration of adjuvant treatment and often do not complete their treatment, missing out on the significant local and survival benefits of radiotherapy.

Hypofractionated radiotherapy (HFRT) delivering higher dose per fraction (2.6-3 Gy per fraction) with lower total dose (around $40 \mathrm{~Gy}$ ) in a shorter duration of time (about 3 weeks) appears a logistically plausible option for patients and also radiotherapy establishments in resource constrained regions like ours. Results of studies by Whelan et al. ${ }^{6}$, Owen et al. ${ }^{7}$ and START Trialists $^{8,9,10}$ groups have proven the equivalent safety and effectiveness of HFRT compared to CFRT in early breast cancer. But in India, most patients present with locally advanced cancer ${ }^{11}$ and mostly undergo mastectomy. There is lack of strong evidence supporting HFRT in post mastectomy patients and hence there is a need to evaluate its safety and effectiveness in locally advanced breast cancer.

Our study, prospectively evaluates the acute toxicities and compliance to radiotherapy among locally advanced breast cancer patients treated with conventional versus hypofractionated radiotherapy following mastectomy.

\section{Materials and Methods}

This study was carried out in a Regional Cancer Center located in the North Eastern region of India. Prior approval from the Institutional Ethics Committee was obtained and performed in accordance with the principles embodied in the Declaration of Helsinki. Informed consent was obtained from each study participant prior to enrolment. This study was registered retrospectively with the Clinical Trial RegistryIndia vide registration no. CTRI/2018/04/013174

\section{Patients}

Histologically proven female breast cancer patients who had undergone modified radical mastectomy and planned for adjuvant radiotherapy as per the Institutional Tumour Board decision, were eligible for enrolment. Patients with age > 18 years, tumour with pathological stage II-III, or patients who underwent neoadjuvant chemotherapy with normal haematological, cardiac and pulmonary functions were considered for accrual. Patients with separation of tangential beam margins (distance between midline and mid-axillary line) more than $22 \mathrm{~cm}$ were excluded. Patients with non-epithelial malignancies (sarcoma, lymphoma), those with co-existent or previous history of other malignancy, those who received prior radiotherapy to chest region and those with severe physical or mental co-morbidities were excluded from the study.

\section{Procedure}

Enrolled patients were randomized on 1:1 basis between the two arms: Arm A (STUDY ARM)- 
Hypofractionation (HF) and Arm B (CONTROL ARM)- Conventional fractionation (CF). Patients in the $\mathrm{HF}$ arm received $267 \mathrm{cGy}$ per fraction to a dose of 40Gy/15 fractions in 3 weeks while those in $\mathrm{CF}$ arm received $200 \mathrm{cGy}$ per fraction to a dose of $50 \mathrm{~Gy} / 25$ fractions in 5 weeks time. Radiotherapy was delivered in once daily fractions, five days a week from Monday to Friday. Patients were treated using 6MV photons in a linear accelerator.

Radiotherapy was delivered to the chest wall using two parallel opposed tangential beams with the patient lying supine on a breast board. 2D simulator based planning was implemented. The cranial margin of the field placed at the level of suprasternal notch, caudal margin $2 \mathrm{~cm}$ below opposite inframammary fold, medial border at midline, lateral border at mid axillary line and anterior border $2 \mathrm{~cm}$ above the highest point of the chest wall. Care was taken to include the mastectomy scar within the treatment volume and the central lung distance less than $2.5 \mathrm{~cm}$. The chest wall contour was replicated and transferred to a digitizer in the treatment planning system to calculate the depth of prescription and check for adequacy of target volume coverage and homogeneity.

For patients whose post mastectomy histopathology reported four or more positive axillary lymph nodes, nodes with gross extracapsular extension or in patients with inadequate axillary dissection (less than 10 number), a separate direct anterior field was used to treat the axilla and supraclavicular region. Dose and fractionation was similar to that received by the chest wall. A gap junction of $0.5 \mathrm{~cm}$ from the chest wall field was used to prevent overlapping of field borders. The superior divergence of the tangential fields was accounted for by using appropriate couch rotation and collimation. No separate fields for treating the internal mammary nodes or posterior axillary boost was employed in any patient.

Radiation toxicities were assessed weekly during radiotherapy and at treatment completion using the RTOG Acute Radiation Morbidity Scoring Criteria $^{12}$. Any treatment gaps during radiotherapy were noted and the cause ascertained.

\section{Study Objectives}

The primary objective of the study was to evaluate safety of hypofractionation and hence to check for radiation induced acute normal tissue toxicities in both the arms. The secondary objective was to compare the compliance between patients in each arm by noting the interruptions in treatment and delay in overall treatment time.

\section{Statistical Analysis}

Data analysis was done using Graph Pad Prism software. Chi-square test for independence and Fischer's exact tests were used for analysis of categorical data while continuous data were analysed using Unpaired t-test, as found appropriate. Where appropriate, 95\% confidence interval (CI) was computed and the alpha level of statistical significance ( $p$-value) was taken as 0.05 .

\section{Results}

From June 2014 to January 2015, 50 patients were accrued and randomized to the two arms of the study -25 in each arm.

The median age of patients in HF arm was 43.04 years versus 48.56 years in the $\mathrm{CF}$ arm. Most of the patients had an ECOG performance score 1 in both the study arms and the number of premenopausal or postmenopausal subjects were evenly distributed in both the study arms (Table $1)$.

All cases in the HF arm had infiltrating duct carcinoma (IDC) while CF arm had 92\% IDC and $8 \%$ mucinous carcinoma. Grade 2 tumours were higher in the $\mathrm{CF}$ arm (52\%), while the $\mathrm{HF}$ arm had more Grade 3 tumours (52\%). The clinical stage of disease was found evenly matched between the two arms. The number of hormone receptor positive patients was higher in the CF arm (Table 2).

With regards to systemic therapy, all except one ptient in the HF arm, received some form of combination chemotherapy. Fifty two percent patients in $\mathrm{HF}$ arm and $56 \%$ in $\mathrm{CF}$ arm received 
Taxanes. Most patients received adjuvant chemotherapy and equal proportion of patients received hormonal therapy in both the arms (Table 3).

Statistical analysis of these data revealed no significance ( $p$-value >0.05) and thus the two study arms were homogenous with regards to the patient, tumour and treatment related characteristics.

The mean chest wall separation and the average central lung distance of the patients in both the arms were comparable. Proportion of patients receiving radiotherapy to nodal regions $(76 \%$ in $\mathrm{HF}$ and $84 \%$ in $\mathrm{CF}$ ) was also found to be similar in both the arms. However, there was a noticeable difference in treatment interruptions and delay in treatment completion between the two arms. It was seen that the 3 week HFRT was completed on an average in 22.8 days while the 5 week CFRT had a mean overall treatment time of 39.76 daysa mean delay of 1.8 days in $\mathrm{HF}$ arm versus 4.76 days in CF arm (p- value 0.0009). Thus, interruptions in treatment completion among patients in the shorter duration HF arm was significantly less (Table 4).
At treatment completion, dermatitis in the irradiated area and odynophagia were the noticeable acute toxicities. There was some grade of dermatitis in every patient undergoing radiotherapy across both the arms. Patients in the HF arm experienced higher rates of Grade 1 dermatitis consisting of follicular or dull erythema and/or dry desquamation when compared to $\mathrm{CF}$ arm (84\% vs $64 \%$ ). Grade 2 dermatitis of tender, bright erythema and/or patchy moist desquamation was higher in the $\mathrm{CF}$ arm compared to $\mathrm{HF}$ arm (28\% vs $16 \%)$. Two patients (8\%) in $\mathrm{CF}$ arm had Grade 3 dermatitis with confluent, moist desquamation of irradiated skin resulting in treatment breaks but no such toxicity occurred in $\mathrm{HF}$ arm. Odynophagia was noticeable exclusively in those patients receiving separate anterior field to treat the axilla and supraclavicular region as per indication. It manifested as mild pain on deglutition relieved on topical anaesthetics and non-narcotic analgesics (Grade 1) with higher incidence among patients of CF arm than HF arm (68\% vs $52 \%$ ). No Grade $4-5$ acute toxicities were found in any patient. (Table 5).

Table 1: Comparison of Patient characteristics.

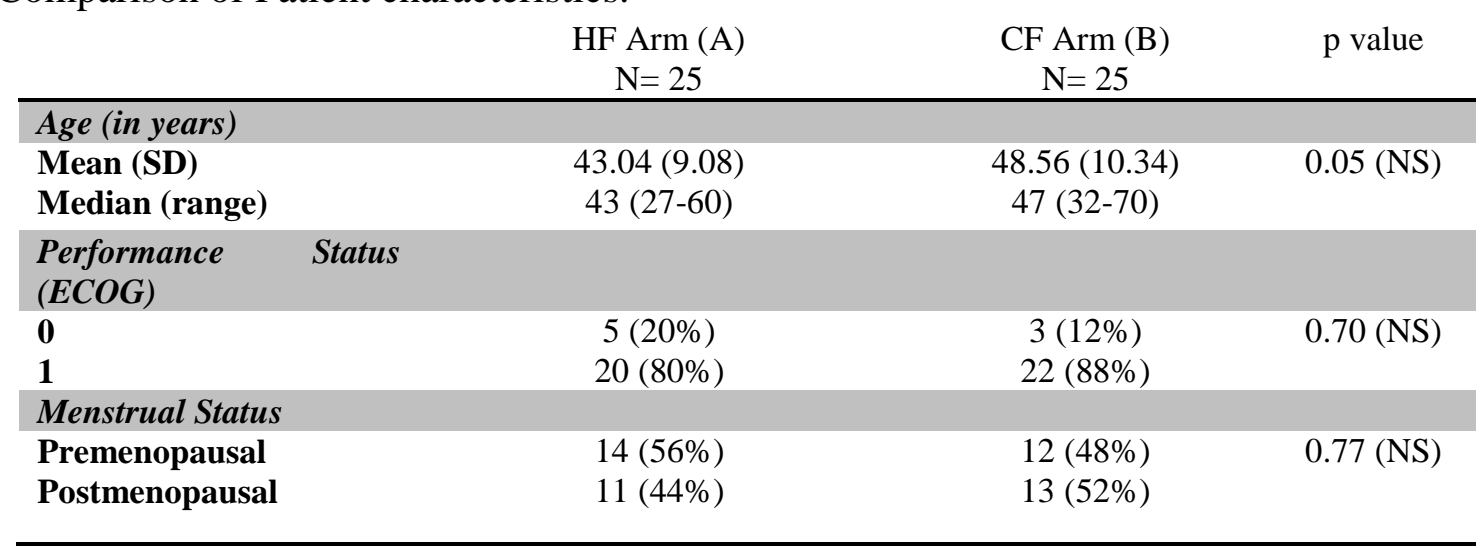


Table 2: Comparison of Tumour characteristics.

\begin{tabular}{|c|c|c|c|}
\hline & $\begin{array}{c}\text { HF Arm (A) } \\
\quad \mathrm{N}=25\end{array}$ & $\begin{array}{c}\text { CF Arm (B) } \\
\mathrm{N}=25\end{array}$ & $\mathrm{p}$ value \\
\hline \multicolumn{4}{|l|}{ Clinical Stage } \\
\hline I & 0 & 0 & \multirow{5}{*}{$0.95(\mathrm{NS})$} \\
\hline IIA & $5(20 \%)$ & $4(16 \%)$ & \\
\hline IIB & $10(40 \%)$ & $9(36 \%)$ & \\
\hline IIIA & $7(28 \%)$ & $8(32 \%)$ & \\
\hline IIIB & $3(12 \%)$ & $4(16 \%)$ & \\
\hline \multicolumn{4}{|l|}{ Histopathology } \\
\hline Infiltrating Duct Ca & $25(100 \%)$ & $23(92 \%)$ & \multirow{2}{*}{0.49 (NS) } \\
\hline Other & 0 & $2(8 \%)$ & \\
\hline \multicolumn{4}{|l|}{ Tumour Grade } \\
\hline 1 & 0 & 0 & \multirow{3}{*}{$0.29(\mathrm{NS})$} \\
\hline 2 & $12(48 \%)$ & $13(52 \%)$ & \\
\hline 3 & $13(52 \%)$ & $10(40 \%)$ & \\
\hline Not applicable & 0 & $2(8 \%)$ & \\
\hline \multicolumn{4}{|l|}{ Tumour Size (pT) } \\
\hline pTx & $2(8 \%)$ & $3(12 \%)$ & \multirow{4}{*}{$0.90(\mathrm{NS})$} \\
\hline pT1 (upto $2 \mathrm{~cm}$ ) & $2(8 \%)$ & $2(8 \%)$ & \\
\hline pT2 $(>2 \mathrm{~cm},<5 \mathrm{~cm})$ & $14(56 \%)$ & $15(60 \%)$ & \\
\hline pT3 $(>5 \mathrm{~cm})$ & $7(28 \%)$ & $5(20 \%)$ & \\
\hline \multicolumn{4}{|c|}{ Lymph node status $(p N)^{*}$} \\
\hline pNo (0) & $8(32 \%)$ & $5(20 \%)$ & \multirow{4}{*}{$0.62(\mathrm{NS})$} \\
\hline pN1a (1-3) & $10(40 \%)$ & $9(36 \%)$ & \\
\hline pN2a (4-9) & $5(20 \%)$ & $7(28 \%)$ & \\
\hline pN3a ( 10 or more) & $2(8 \%)$ & $4(16 \%)$ & \\
\hline \multicolumn{4}{|l|}{ Estrogen Receptor } \\
\hline Positive & $14(56 \%)$ & $16(64 \%)$ & \multirow[t]{2}{*}{$0.77(\mathrm{NS})$} \\
\hline Negative & $11(44 \%)$ & $9(36 \%)$ & \\
\hline \multicolumn{4}{|l|}{ Progesterone Receptor } \\
\hline Positive & $11(44 \%)$ & $13(52 \%)$ & \multirow[t]{2}{*}{$0.77(\mathrm{NS})$} \\
\hline & $14(56 \%)$ & $12(48 \%)$ & \\
\hline \multicolumn{4}{|l|}{ Her 2 neu expression } \\
\hline Positive & $7(28 \%)$ & $8(32 \%)$ & \multirow[t]{2}{*}{$1.0(\mathrm{NS})$} \\
\hline Negative & $18(72 \%)$ & $17(68 \%)$ & \\
\hline
\end{tabular}

Table 3: Comparison of Systemic therapy characteristics.

\begin{tabular}{lccc} 
& HF Arm (A) & CF Arm (B) & p value \\
\hline Chemotherapy & & N=25 & \\
None & $1(4 \%)$ & 0 & \\
Neo-Adjuvant only & $4(16 \%)$ & $6(24 \%)$ & $0.40(\mathrm{NS})$ \\
Neo-Adjuvant $\rightarrow$ Adjuvant & $3(12 \%)$ & $6(24 \%)$ & \\
Adjuvant only & $17(68 \%)$ & $13(52 \%)$ & \\
Chemotherapy Agent & $1(4 \%)$ & $1(4 \%)$ & \\
AC & $6(24 \%)$ & $4(16 \%)$ & \\
FAC & $4(16 \%)$ & $6(24 \%)$ & \\
FEC & $3(12 \%)$ & $4(16 \%)$ & \\
TAC & $4(16 \%)$ & $3(12 \%)$ & \\
AC $\rightarrow$ T & $6(24 \%)$ & $7(28 \%)$ & \\
FEC $\rightarrow$ T & & & \\
Hormonal Therapy & $10(40 \%)$ & $9(36 \%)$ & \\
None & $7(28 \%)$ & $8(32 \%)$ & \\
Tamoxifen & $8(32 \%)$ & $8(32 \%)$ & \\
Aromatase Inhibitors & & & \\
\hline
\end{tabular}


Table 4: Comparison of Radiotherapy characteristics between the study arms

\begin{tabular}{lccc} 
& $\begin{array}{c}\mathrm{HF} \text { Arm }(\mathrm{A}) \\
\mathrm{N}=25\end{array}$ & $\begin{array}{c}\mathrm{CF} \text { Arm }(\mathrm{B}) \\
\mathrm{N}=25\end{array}$ & p value \\
\hline $\begin{array}{l}\text { Chest Wall separation } \\
\text { (in } \text { cm) }\end{array}$ & & & \\
$\begin{array}{l}\text { Mean (SD) } \\
\text { Median (Range) }\end{array}$ & $18.28(1.77)$ & $18.64(1.99)$ & $0.50(\mathrm{NS})$ \\
$\begin{array}{l}\text { Central Lung Distance } \\
\text { (in cm) }\end{array}$ & $18(16-22)$ & $18(15-22)$ & \\
Mean (SD) & & & $0.90(\mathrm{NS})$ \\
Median (Range) & $1.82(0.5752)$ & $1.84(0.5346)$ & $0.73(\mathrm{NS})$ \\
Radiotherapy to SCF/Axilla & $2.0(1.0-2.5)$ & $2.0(1.0-2.5)$ & \\
Yes & $19(76 \%)$ & $21(84 \%)$ & $4(16 \%)$ \\
No & $6(24 \%)$ & & $<0.0001 *$ \\
RT overall treatment time (in & & & \\
days) & & $39.76(3.778)$ & \\
Mean (SD) & $22.8(1.826)$ & $39(35-51)$ & \\
Median (Range) & $22(21-27)$ & &
\end{tabular}

\begin{tabular}{|c|c|c|c|}
\hline \multicolumn{4}{|c|}{$\begin{array}{l}\text { Interruptions/ Delays in } R T \\
\text { completion (in days) }\end{array}$} \\
\hline Mean (SD) & $1.8(1.826)$ & $4.76(3.778)$ & $0.0009 *$ \\
\hline Median (Range) & $1(0-6)$ & $4(0-16)$ & \\
\hline
\end{tabular}

Table 5: Comparison of Radiotherapy induced Acute toxicities.

\begin{tabular}{lccc} 
& HF Arm (A) & CF Arm (B) & p value \\
& $\mathrm{N}=25$ & $\mathrm{~N}=25$ & \\
\hline Dermatitis & & 0 & $0.17(\mathrm{NS})$ \\
Grade 0 & 0 & $16(64 \%)$ & \\
Grade 1 & $21(84 \%)$ & $7(28 \%)$ & \\
Grade 2 & $4(16 \%)$ & $2(8 \%)$ & $0.39(\mathrm{NS})$ \\
Grade 3 & 0 & $8(32 \%))$ & $17(68 \%)$ \\
Odynophagia (Esophagitis) & $12(48 \%)$ & & \\
None (Grade 0) & $13(52 \%)$ & & \\
Mild (Grade 1) & & & \\
\hline
\end{tabular}

\section{Discussion}

Postmastectomy adjuvant radiotherapy improves loco-regional control and overall survival in breast cancer patients as shown in various randomized trials $^{2,3,4}$ and meta analysis ${ }^{5}$. Hypofractionation in delivering radiotherapy for breast cancer is now an established protocol, at least in treating early stages.

Whelan et al. ${ }^{6}$, Owen et al. ${ }^{7}$ and the START Trials $\mathrm{A}^{8}$ and $\mathrm{B}^{9}$ have shown in their results that the local-regional relapse did not differ significantly between conventional fractionation and any of the hypofractionated regimens evaluated while the normal tissue effects like breast shrinkage, edema and telengiectasia were significantly less in the hypofractionated arms. Qi et al. ${ }^{13}$ applied the Linear Quadratic model and Poisson statistics on the data obtained from these four RCTs and confirmed the radiobiological basis of the results by establishing that breast cancer cells have a low $\alpha / \beta$ (range: 0.75-5.01Gy) and hence hypofractionation may be more effective than conventional fractionation in treating breast cancer with radiotherapy.

However, the patients in the START Trials consisted of early breast cancers and only a small proportion of them underwent mastectomy $(15 \%$ in START $\mathrm{A}^{8}$ and $8 \%$ in START $\mathrm{B}^{9}$ ). Hypofractionated radiotherapy for PMRT in locally advanced breast cancer has not been evaluated in many randomized control trials. Pinitpatcharalert A et al. ${ }^{14}$ reported significant increase in 5-year OS among the patients receiving HFRT (2.65Gy x 16-18 fractions) with 
no difference in acute and late toxicities, in his retrospective study of 215 patients. Similar results were reported by Elsayed et al. ${ }^{15}$ and Sun et al. ${ }^{16}$ in their retrospective studies. Elsayed et al. ${ }^{17}$, in another prospective trial of 47 patients receiving PMRT with conventional versus hypofractionation $(42.72 \mathrm{~Gy} / 16 \mathrm{~F})$ found equivalent OS, DFS and adverse effects and concluded that HFRT is advantageous as it reduced workload and cost of treatment.

Our study was a single institution, hospital based, prospective, randomised study evaluating HFRT versus CFRT following mastectomy, in intermediate-high risk breast cancer patients. Both the study arms were well matched statistically with regards to the patient, tumour and treatment related prognostic factors. Also our baseline study characteristics were quite comparable to those of Sun et al. ${ }^{16}$ and Elsayed et al. ${ }^{17}$.

Our study results show HFRT to be safe and well tolerated among postmastectomy patients. Acute dermatitis was less in severity in the HFRT arm (Table 5) and did not result in treatment breaks unlike that in the CFRT arm. Our findings are in agreement with that reported by Pinitpatcharalert A et al. ${ }^{14}$, Elsayed et al. ${ }^{15}$ and Sun et al. ${ }^{16}$ in terms of adverse events related to radiotherapy.

A significant observation of our study was that radiotherapy interruptions were higher in CFRT (mean 4.8 days, $\mathrm{SD}=3.778$, range: 0 -16) delivered in 5 weeks compared to the 3 weeks treatment of HFRT (mean 1.8 days, $\mathrm{SD}=1.826$, range: 0-6) - a p-value of 0.0009 . Similar results were shown by Elsayed et al. ${ }^{17}$ also, in his study. In our study, 2 patients $(8 \%)$ in the $\mathrm{CF}$ arm developed wet desquamation of skin (Grade 3 dermatitis) and suffered forced treatment breaks of 10 days and 16 days respectively, while none in the $\mathrm{HF}$ arm suffered such consequences. But there were other reasons also for treatment interruptions among our patients- the most frequent being logistics issues (like finance, transportation etc.) and technical problems in the radiotherapy treatment facility (machine breakdown and maintenance etc.). Longer the treatment duration of radiotherapy extends, more will be the chances of these factors delaying treatment completion and more the treatment dropout. Thus, adopting a 3 week HFRT against a 5 week CFRT regime in routine clinical practice will likely improve treatment compliance among patients and also optimize utilization of already constrained resources of radiotherapy in overburdened centers like ours.

Major limitations of this study are the relatively less number of patients accrued and lack of analysis for late toxicities. The long term results of the trial with a longer follow up are awaited to evaluate the effectiveness of our HFRT regimen and the associated late toxicities.

\section{Conclusion}

Hypofractionated radiotherapy in postmastectomy patients of locally advanced breast cancer was an area of debate among radiation oncologists. But with the emergence of results from recent randomized trials, it can now be emphasized strongly that HFRT in postmastectomy patients is as safe as CFRT and can be used in routine clinical practice. A shorter duration treatment is more economical for the patients and logistically convenient for treatment providers.

\section{Acknowledgement}

The authors would like to acknowledge the support of the senior and junior residents of Radiation Oncology department, BBCI for their continued help and support.

\section{Sources of Fund: None}

\section{References}

1. GLOBOCAN 2012- Breast Cancer Estimated Incidence, Mortality and Prevalence Worldwide in 2012. Available at:

http://globocan.iarc.fr/Pages/fact_sheets_c ancer.aspx

2. Overgaard M, Nielsen HM, Overgaard J. Is the benefit of postmastectomy irradiation limited to patients with four or 
more positive nodes, as recommended in international consensus reports? A subgroup analysis of the DBCG 82 b\&c randomized trials. Radiother Oncol. 2007;82(3):247-53.

3. Overgaard M, Hansen PS, Overgaard J, et al. Postoperative radiotherapy in high-risk premenopausal women with breast cancer who received adjuvant chemotherapy. Danish Breast Cancer Cooperative Group 82b Trial. N Engl J Med. 1997;337(14):949-55.

4. Overgaard M, Jensen MB, Overgaard J, et al. Postoperative radiotherapy in high-risk postmenopausal breastcancer patients given adjuvant tamoxifen: Danish Breast Cancer Cooperative Group DBCG 82c randomized trial. Lancet. 1999;353(9165):1641-8.

5. Early Breast Cancer Trialists' Collaborative Group, Darby S, McGale P, et al. Effect of radiotherapy after breastconserving surgery on 10-year recurrence and 15-year breast cancer death: metaanalysis of individual patient data for 10,801 women in 17 randomized trials. Lancet 2011;378(9804):1707-16. doi: 10.1016/S0140-6736(11)61629-2.

6. Whelan TJ, Pignol JP, Levine MN, et al. Long-term results of hypofractionated radiation therapy for breast cancer. $\mathrm{N}$ Engl J Med. 2010;362(6):513-20. doi: 10.1056/NEJMoa0906260.

7. Owen JR, Ashton A, Bliss JM, et al. Effect of radiotherapy fraction size on tumor control in patients with early stage breast cancer after local tumor excision: longterm results of a randomized trial. Lancet Oncol. 2006 7(6):467-71.

8. START Trialists' Group, Bentzen SM, Agrawal RK, Aird EG, Barrett JM, et al. The UK Standardisation of Breast Radiotherapy (START) Trial A of radiotherapy hypofractionation for treatment of early breast cancer: a randomised trial. Lancet Oncol 2008; 9: 331-341. doi: $10.1016 / \mathrm{S} 1470$ 2045(08)70077-9

9. START Trialists' Group, Bentzen SM, Agrawal RK, Aird EG, Barrett JM, et al. The UK Standardisation of Breast Radiotherapy (START) Trial B of radiotherapy hypofractionation for treatment of early breast cancer: a randomised trial. Lancet. 2008 371(9618): 1098-107. doi: 10.1016/S01406736(08)60348-7.

10. Joanne S Haviland, J Roger Owen, John A Dewar, Rajiv K Agrawal et al. The UK Standardisation of Breast Radiotherapy (START) trials of radiotherapy hypofractionation for treatment of early breast cancer: 10-year follow-up results of two randomised controlled trials. Lancet Oncol 2013; 14(11): 1086-94. doi.org/10.1016/S1470-2045(13)70386-3

11. Saxena S, Rekhi B, Bansal A, Bagga A, Chintamani, Murthy NS: Clinicomorphological patterns of breast cancer including family history in a New Delhi hospital, India - a cross-sectional study. World J Surg Oncol 2005;3:67. doi: 10.1186/1477-7819-3-67

12. Cox JD, Stetz J, Pajak TF. Toxicity criteria of the Radiation Therapy Oncology Group (RTOG) and the European Organization for Research and Treatment of Cancer (EORTC). Int J Radiat Oncol Biol Phys 1995; 31(5): 1341-6. doi: 10.1016/03603016(95)00060-C

13. Qi XS, White J, Li XA. Is $\alpha / \beta$ for breast cancer really low? Radiother Oncol. 2011;100(2):282-88.

DOI: https://doi.org/10.1016/j.radonc.2011 .01 .010

14. Pinitpatcharalert A, Chitapanarux I et al. A retrospective study comparing hypofractionated radiotherapy and conventional radiotherapy in 
postmastectomy breast cancer. J Med Assoc Thai 2011; 94 Suppl 2:S94-102.

15. El-Sayed MA and Abdel-Wanis. Comparison of Hypofractionated and Conventional Radiotherapy Protocols in Breast Cancer Patients: A Retrospective Study. J Cancer Sci Ther 2012; 4: 158163. doi: 10.4172/1948-5956.1000132

16. GY Sun, SL Wang, YW Song et al. Hypofractionated Radiation Therapy After Mastectomy for the Treatment of HighRisk Breast Cancer: 5-Year Follow-Up Result of a Randomized Trial. Int J Radiat Oncol Biol Phys 2017; 99(2): S3-S4

17. Elsayed MA, Magdy KAA. Postmastectomy Hypofractionation

Radiotherapy in Breast Cancer Patients. Cancer and Oncology Research 2014; 2(7): 87-93. DOI: 10.13189/cor.2014.020701. 